Vincent Hoffmann-Martinot

\title{
Zentralisierung und Dezentralisierung in Frankreich
}

\section{Der Staat und die Gebietskörperschaften}

Die politisch-administrative Struktur Frankreichs war fast zwei Jahrhunderte lang durch einen pyramidalen, streng zentralistischen Charakter geprägt, der durch die Französische Revolution und die Reformen Napoleons geschaffen worden war. Am Ende des 20. Jahrhunderts aber hat sich Frankreich weit von diesem napoleonischen Modell entfernt. Heute erinnert das komplexe Nebeneinander von Gebietskörperschaften und anderen Institutionen ebenso wie die Überschneidung der Zuständigkeitsbereiche auf seltsame Weise an die unübersichtliche Situation des vorrevolutionären Frankreich (Ancien Régime). Der Staat ist keine große ordnende Kraft mehr. Seit den sechziger bzw. siebziger Jahren ist er unablässig dabei zu delegieren, zu dekonzentrieren und $\mathrm{zu}$ dezentralisieren. Eine Unzahl von öffentlichen, halböffentlichen, ja privaten Akteuren bildet die institutionelle Struktur Frankreichs, dessen Devise gleichwohl "die unteilbare Republik" bleibt"

\section{Die verschiedenen öffentlichen Akteure}

Grundsätzlich ist die Verwaltungsstruktur Frankreichs von zwei Akteursgruppen mit unterschiedlichem Status geprägt. Dem Zentralstaat stehen drei Gebietskörperschaften gegenüber (vgl. Abb. 1):

- 26 Regionen (régions), davon 22 im Mutterland, 4 in Übersee; 
- 100 Departements (départements), davon 96 im Mutterland, 4 in Übersee;

- 36763 Gemeinden (communes), davon 36551 im Mutterland, 212 in Übersee.

Der Zentralstaat ist nicht nur in der Hauptstadt Paris mit seinen Ministerien präsent, sondern durch die nachgeordneten Dienststellen der Zentralverwaltung auch im gesamten französischen Territorium. Hier spielen vor allem die regionalen bzw. departementalen Direktionen der Ministerien (etwa die departementale Baubehörde, Direction départementale de l'équipement, oder die Landwirtschaftsbehörde, Direction départementale de l'agriculture) sowie der von der Regierung eingesetzte Präfekt als Repräsentant des Staates im Departement bzw. in der Region eine Rolle.

Diesen nachgelagerten staatlichen Behörden stehen als eigene Akteure die drei Gebietskörperschaften (collectivités territoriales) mit ihren eigenen Verwaltungen gegenüber. Diese stehen nebeneinander; so ist die Region nicht etwa den Departements oder den Kommunen gegenüber weisungsberechtigt. 
Der Staat ist nach wie vor der größte Arbeitgeber. Er beschäftigt 2,2 Mio. Personen, während die Gebietskörperschaften 1,4 Mio. und die öffentlichen Krankenhäuser 0,8 Mio. Bedienstete zählen ${ }^{2}$. Sein Zuständigkeitsbereich ist groß, da die verschiedenen Pariser Ministerien neben den klassischen hoheitsrechtlichen Funktionen (Inneres, Wirtschaft, Finanzen, Verteidigung, Äußeres usw.) auch mit zahlreichen anderen Bereichen betraut sind, z.B. Soziales, Raumordnung, Landwirtschaft, Jugend und Sport oder auch Kultur. Im Unterschied zum Zusammenwirken von Bundes- und Landesverwaltung in der Bundesrepublik lenkt die Zentralregierung von der Hauptstadt aus die gesamte staatliche Verwaltung; die Ministerien verfügen über zahlreiche, gut ausgestattete nachgeordnete Dienststellen auf regionaler und lokaler Ebene. Der von der Regierung ernannte Präfekt ist das Symbol schlechthin dieser territorialen Verankerung des Staates. Als Vertreter des Staates im Departement bzw. in der Region verkörpert er die Einheit der Nation. Er wacht über den Vollzug der Regierungspolitik und koordiniert die Tätigkeit der verschiedenen nachgeordneten Dienststellen des Staates. Diese staatliche Kontrolle der territorialen Ebene wird von ihren Verfechtern in der Regel als notwendig bezeichnet, um angesichts der extremen territorialen Zersplitterung des französischen Verwaltungssystems die Einheitlichkeit des Gesetzesvollzuges und darüber hinaus den territorialen Zusammenhalt des Landes zu garantieren. 


\section{a) Kommunen}

In keinem Land Europas ist die Anzahl der Kommunen so hoch wie in Frankreich: 36551 im Mutterland, 36763 einschließlich der überseeischen Gebiete. Diese Zahl ist doppelt so hoch wie in Deutschland (16 061). Auch hinsichtlich der Anzahl der kommunalen Mandatsträger - immerhin 500000 Bürger - stellt Frankreich die übrigen europäischen Länder in den Schatten. 80 Prozent der Kommunen (hauptsächlich Landgemeinden) haben weniger als 1000 Einwohner, und bei lediglich 2 Prozent liegt die Einwohnerzahl über $10000^{3}$. Die Gemeinden sind für alle kommunalen Angelegenheiten zuständig. Sie sind verantwortlich für den Städtebau, für den Bau und Unterhalt der Vor- und Grundschulen sowie für Sporteinrichtungen, für das kommunale Straßen- bzw. Wegenetz und für die städtischen Transportmittel (vgl. Tab. 1). Zudem nehmen sie verschiedene Funktionen im Namen des Staates wahr (insbesondere Standesamtsregister und Wählerverzeichnisse).

Die territoriale Neuordnung ist ebenso wie die Rationalisierung des französischen Verwaltungsraums ein altes Vorhaben, das sich bis zur Französischen Revolution 1789 zurückverfolgen läßt. Aber erst in den sechziger Jahren unseres Jahrhunderts, vor dem Hintergrund tiefgreifender sozioökonomischer Umwälzungen und einer rapiden Urbanisierung, erschien sie wirklich auf der politischen Tagesordnung. Am 16. Juli 1971 wurde ein Gesetz zur kommunalen Neugliederung verabschiedet. Die Bilanz seiner Anwendung muß allerdings als ausgesprochen mager bezeichnet werden. Zwischen 1971 und 1974 kam es nur zu 804 Zusammenlegungen von insgesamt 1972 Kommunen, und es wurde ein einziger Stadt-Umland-Verband (Brest) geschaffen. Die meisten Eingemeindungen betrafen lediglich zwei oder drei kleine Orte. Dieser Mißerfolg ist auf mangelnde Unterstützung von Seiten der Bevölkerung der betreffenden Kommunen, vor allem aber auf das von Pierre Grémion als "periphere Macht" bezeichnete Zusammenspiel zwischen Vertretern des 
Zentralstaates und gewählten öffentlichen Notabeln zurückzuführen: "Beamte auf territorialer Ebene und gewählte örtliche Repräsentanten sind Mitglieder desselben Systems von Honoratiorenmacht. Ein subtiles Spiel von Konfliktaustragung und Komplizenschaft bindet sie aneinander. Gewählte und Bürokraten sind aufeinander angewiesen, wenn es darum geht, die öffentlichen Angelegenheiten $\mathrm{zu}$ verwalten. Ihre gegenseitige Abhängigkeit kann so weit gehen, daß sich ihre Rollen durchdringen." ${ }^{4}$

Der Mißerfolg der territorialen Neugliederung ist ein ausgezeichnetes Beispiel für diesen dominierenden Regulierungsmodus des politischadministrativen Systems im ländlichen, traditionellen Frankreich, der auf der Zweierkonstellation zwischen dem Präfekt und "seinen" Notabeln beruht ${ }^{5}$. Seither haben es alle Regierungen, egal welcher politischer Couleur, sorgfältig vermieden, auch nur die geringste Reform einzuleiten, die auf eine territoriale Neuordnung abzielt, da ihnen das politische Risiko $\mathrm{zu}$ hoch erschien.

Die Regierungen verfolgten deshalb seit den achtziger Jahren viel bescheidenere Ziele. Sie gingen vom Bestehenden aus und versuchten, die gemeindeübergreifende Kooperation durch Überzeugungsarbeit und nicht durch Zwang zu verstärken. So wurde die vertragliche Regelung der Beziehungen zwischen Gebietskörperschaften sowie die Schaffung kommunenübergreifender Organisationsformen gefördert. Im Gegensatz zur früheren Praxis obliegen Initiative und Durchführung der Maßnahmen nun hauptsächlich den örtlichen Akteuren, nicht der staatlichen Verwaltung. Dieser Dezentralisierungsansatz beruht ausschließlich auf der Kooperationsbereitschaft der lokalen Akteure. Er wurde durch ein Gesetz vom Januar 1983 eingeleitet, das die Erarbeitung gemeindeübergreifender Vereinbarungen ("Charten") zur gemeinsamen Entwicklung und Raumplanung vorsieht. Diese erlauben es den Kommunen, Vereinbarungen zur gemeinsamen Planung der gesamten lokalen Entwicklung zu treffen. 
Diese Charten haben sich rasch ausgebreitet, denn 1985 war bereits jede sechste Kommune dabei, eine solche Charta vorzubereiten oder umzusetzen, und zwar in geographisch wie soziologisch gesehen sehr unterschiedlichen Milieus: ländliche Gebiete, Täler, Gebirgsgegenden, Kleinstädte mit ihrem Einzugsgebiet, Vorstädte, Ballungszentren usw. Den größten Erfolg hatten die Charten in den ländlichen Gebieten, wo Kleinstädte zwischen 5000 bis 10000 Einwohner eine Schlüsselrolle spielten. Diese voluntaristische Dynamik ist durch das territoriale Rahmengesetz vom 6. Februar 1992 bestätigt worden. Es schafft in jedem Departement einen Ausschuß für kommunenübergreifende Zusammenarbeit, dessen Aufgabe es ist, eine Kooperation zu fördern, die "auf dem freien Willen der Kommunen beruht, innerhalb von Solidaritätsgebieten gemeinsame Entwicklungsprojekte zu erarbeiten" (Art. 66).

Nach dem gescheiterten Neugliederungsversuch von 1971 ist es zu zahlreichen Kooperationsformen zwischen Gebietskörperschaften gekommen: Zwischen 1972 und 1995 ist die Anzahl der interkommunalen Zweckverbände mit einer Aufgabe (syndicats intercommunaux à vocation unique/ SIVU) von 9289 auf 14490 angestiegen, die der Zweckverbände mit mehrfachen Aufgaben (syndicats intercommunaux à vocation multiple/ SIVOM) von 1243 auf 2298 und die der Stadt-Umland-Verbände (districts) von 95 auf 318. Die weniger Handlungsspielraum lassende Kooperationsform der Stadt-Umland-Verbände in großstädtischen Ballungszentren (communautés urbaines) hat weniger Zuspruch gefunden (1972: 9, 1995: 10). In den neunziger Jahren sind mit den 894 Gemeindeverbänden (communautés de communes) und den 4 Stadtverbänden (communautés de villes) zusätzliche Strukturen der Zusammenarbeit entstanden. Schließlich können sich die Kommunen im Rahmen von Mischverbänden (syndicats mixtes) auch mit anderen 
Gebietskörperschaften oder aber mit öffentlichen bzw. privaten Organisationen zusammenschließen (1995: 1 100).

\section{b) Departement}

Die zweite grundlegende Gebietskörperschaft ist das Departement, in dessen Zuständigkeitsbereich die Verwaltung der Sozialhilfe, Bau und Unterhalt der Schulen der Sekundarstufe I (collèges) sowie der überörtliche Schülertransport fallen. 1994 haben die Departements insgesamt $222 \mathrm{Mrd}$. Francs ausgegeben ${ }^{6}$. Es gibt 100 Departements, darunter 4 überseeische. Die Departements sind übergemeindliche Gebietskörperschaften (ähnlich den Kreisen in Deutschland), die vor allem für die ländlichen Gebiete von Bedeutung sind. Die Hauptaufgabe des Departements besteht nämlich darin, die Entwicklung derjenigen Gebiete zu unterstützen, die von den Wirtschaftszentren am weitesten entfernt sind. Im Vergleich zur Situation der Kommunen hält die relativ niedrige Anzahl der Departements die Disparitäten des wirtschaftlichen Potentials in Grenzen. Aber zwischen Paris (2,1 Mio. Einwohner), das sowohl den Status einer Stadt als auch den eines Departements hat, dem Departement Nord (2,5 Mio. Einwohner) sowie den bevölkerungsarmen Departements wie Lozère (73 000) oder Creuse (131 000) liegen Welten. 
c) Region

Die Regionen erhielten mit den Dezentralisierungsgesetzen von 1982 erstmalig den vollwertigen Status einer Gebietskörperschaft. Im französischen Mutterland gibt es 22 Regionen (einschließlich Korsika); die vier überseeischen Departements haben ebenfalls den Status einer Region. Sie sind in der Hauptsache für Raumplanung, Berufsausbildung und Bau sowie Unterhalt der Schulen der Sekundarstufe II (lycées) zuständig. Sie sind darüber hinaus im Hochschulwesen und in der Forschung, bei den öffentlichen Verkehrsmitteln sowie bei der Unterstützung von Unternehmen aktiv. Die Handlungsfelder der Regionen sind sehr viel gezielter und spezialisierter als die der Kommunen und der Departements. Es handelt sich eher um eine projektorientierte Verwaltung (administration de mission) als um eine klassische Verwaltungsbehörde (administration de gestion). An ihrer Dynamik besteht kein Zweifel; sie äußert sich sowohl in der kräftigen Aufstockung ihres Haushalts als auch in den vielfältigen politischen Innovationen, die sie bereits eingeleitet hat. Der Entwicklung der Regionen sind jedoch Grenzen gesteckt, weil sie der wachsenden Konkurrenz der Departements und der bedeutendsten Städte ausgesetzt sind ${ }^{7}$.

\section{Institutionelle Gleichförmigkeit und Machtkonzentration}

Die interne Organisation und die Machtverteilung in den französischen Gebietskörperschaften weisen zwei Hauptmerkmale auf. Erstens ist auf allen Ebenen - von einigen wenigen Ausnahmen abgesehen ${ }^{8}$ - das gleiche Organisationsmodell in Kraft ( $v g l$. Abb. 1 und 2). Zweitens konzentriert sich die Macht beim jeweiligen Chef der Exekutive, gleichgültig ob es sich dabei um den Bürgermeister, den Präsidenten der Departementsverwaltung oder den Präsidenten der Regionalverwaltung handelt. 
Das politische System der französischen Gebietskörperschaften läßt sich als "Bürgermeisterverfassung" bezeichnen. Im Gegensatz zur Süddeutschen Ratsverfassung mit ihrem direkt gewählten Bürgermeister, die sich in Deutschland im Laufe der neunziger Jahre auf fast alle Bundesländer ausgedehnt hat, wird in Frankreich lediglich der Gemeinderat von den Bürgern gewählt und wählt seinerseits den neuen Bürgermeister aus seiner Mitte. Dieser aber beherrscht die kommunalpolitische Szene. Seiner Macht sind wenig institutionelle Schranken gesetzt.

"Das lokale demokratische System funktioniert in Frankreich umgekehrt: Es behauptet, daß der Stadtrat den Bürgermeister wählt; in Wirklichkeit aber ist es der Bürgermeister, der den Stadtrat kooptiert. Es unterscheidet zwischen einer beratenden Versammlung, die entscheidet, und einem ausführenden Organ; häufig aber liegt alle Macht bei der Exekutive, und die Macht des Stadtrats beschränkt sich auf Zustimmung und 'Zurechtweisung'."'

Die Allmacht des Bürgermeisters ${ }^{10}$ - wie auch die des Präsidenten der Departements- bzw. der Regionalverwaltung seit den Dezentralisierungsgesetzen - wird verstärkt durch seine in der Praxis lange Amtsdauer. Zwar muß sich der französische Bürgermeister alle sechs Jahre zur Wiederwahl stellen, aber die Fälle von Dauer-Amtsinhabern sind Legion: z.B. Tony Larue, der erstmals 1935 in Grand-Quevilly Oberbürgermeister wurde und sechzig Jahre lang ohne Unterbrechung wiedergewählt wurde; oder Jacques Chaban-Delmas, Oberbürgermeister von Bordeaux von 1947 bis 1995, der bezeichnenderweise mit dem Spitznamen "Herzog von Aquitaine" versehen wurde. Zwischen 1945 und 1983 betrug die durchschnittliche Amtsdauer der Oberbürgermeister in Städten mit über 15000 Einwohnern neun Jahre; über zwei Drittel dieser Kommunen kannten innerhalb von vierzig Jahren nur zwei oder drei verschiedene Oberbürgermeister ${ }^{11}$. Eine solche praktisch unbegrenzte Langlebigkeit gilt in Frankreich als natürlich und ist bisher nie wirklich 
öffentlich in Frage gestellt oder diskutiert worden - trotz einer zunehmenden Zahl von Korruptionsaffären, die das Problem der Machtbegrenzung der Bürgermeister neu aufwerfen.

In diesem System beruht die Macht der Exekutive auch auf der Unterstützung durch eine ausreichend stabile Mehrheit von Stadträten, deren Herausbildung durch die Anwendung des Mehrheitswahlrechts gefördert wird. In Kommunen mit weniger als 3.500 Einwohnern wird das romanische Mehrheitswahlrecht mit zwei Wahlgängen praktiziert. Die siegreiche Liste erhält alle Sitze. In Gemeinden mit mehr als 3.500 Einwohnern wird ebenfalls nach dem romanischen Mehrheitswahlprinzip in zwei Wahlgänge gewählt. Erreicht eine Liste im ersten Wahlgang die absolute oder im zweiten Wahlgang die relative Mehrheit, so fallen ihr die Hälfte aller Sitze zu. Die restlichen Sitze werden unter allen Listen, die mehr als $5 \%$ der Stimmen aus sich vereinigen konnten, proportional aufgeteilt. Diese Mischung aus Mehrheits- und Verhältniswahlrecht garantiert einerseits regierungsfähige Mehrheiten in den Gemeinderäten und ermöglicht andererseits eine Repräsentation der Minderheitenlisten. Da das Mehrheitswahlrecht eine polasierende Wirkung entfaltet, kommt es in der Regel. zu Listenverbindungen nach dem Rechts-Links-Schema, spätestens nach dem ersten Wahlgang. So werden die meisten Gemeinden von Wahlbündnissen regiert. Die siegreiche Liste verfügt in jedem Fall über eine bequeme Mehrheit: mindestens drei Viertel der Sitze im Fall eines Erfolgs im ersten Wahlgang und z.B. zwei Drittel im Fall einer Liste, die im zweiten Wahlgang nur ein Drittel der abgegebenen Stimmen erhält.

Die extrem starke Position des Chefs der Exekutive in den Gebietskörperschaften wird durch die in Frankreich traditionell verbreitete Ämterhäufung noch verstärkt. Zwei Gesetze vom 30. Dezember 1985 haben jedoch begonnen, der traditionellen Praxis der unbeschränkten Ämterhäufung Grenzen zu ziehen. Von nun an darf niemand mehr als zwei 
der folgenden Mandate gleichzeitig innehaben: Parlamentsabgeordneter, Senator, Europaabgeordneter, Mitglied des Regionalrats, Mitglied des Departementsrats, Mitglied des Pariser Stadtrats, Bürgermeister einer Stadt mit über 20000 Einwohnern und beigeordneter Bürgermeister in Städten mit über 100000 Einwohnern. Die neue Linksregierung, die aus den Parlamentswahlen vom 25. Mai und vom 1. Juni 1997 hervorgegangen ist, hat eine noch restriktivere Gesetzgebung angekündigt, die die parallele Ausübung von einem Parlamentsmandat und einem lokalen Mandat (Stadt, Departement oder Region) oder den Vorsitz in zwei lokalen Exekutivorganen untersagen soll. Bereits bei der ersten Kabinettssitzung der Linksregierung am 4. Juni 1997 hat Premierminister Lionel Jospin daran erinnert, daß die Minister nicht mehr gleichzeitig Funktionen in der lokalen Exekutive ausüben sollten, wie dies bis dahin allgemein akzeptiert und praktiziert wurde. Diese bedeutende Entwicklung der Konzeptionen und Regeln der berufsmäßig betriebenen Politik, die von den meisten Bürgern befürwortet wird, dürfte Ausdruck der unabdingbaren Modernisierung des politischen Lebens Frankreichs sein, das bislang in zu großem Maße durch die lokalen "großen Notabeln" beherrscht wird.

Großstadt-Oberbürgermeister, die aufgrund ihrer Belastung mit anderen politischen Mandaten ihre Stadt nur am Wochenende regieren; Stadtoberhäupter, die sich sogar als Siebzigjährige ohne weiteres zur Wiederwahl stellen können; auf der lokalen Ebene fehlende Beteiligungsrechte wie der Bürgerentscheid: Dies sind die Charakteristika des tradierten Notabelnsystems in Frankreich, das sich von der kommunalpolitischen Praxis in Deutschland erheblich unterscheidet. Allerdings gewinnt das Leitbild eines modernen kommunalen Berufspolitikers zunehmend an Bedeutung. Zwei Faktoren tragen dazu bei: zum einen der Dezentralisierungsproze $\beta$, in dessen Folge der Zugang zur Pariser Zentralmacht für die Mandatsträger sehr viel weniger bedeutsam wird; zum anderen die steigenden Erwartungen und Ansprüche der Bürger 
an die Ergebnisse der kommunalen Politik. Diese Tendenz ist bereits seit den siebziger Jahren auszumachen, als eine neue Generation von Mandatsträgern an die Macht kam. Diese versuchten, die Beziehungen neu zu gewichten, die zwischen ihnen selbst, den Generalisten oder "Dilettanten" (um die Terminologie von Max Weber aufzugreifen) und den "Spezialisten" (d.h. Verwaltungsexperten) existierten. Letztere waren seit langem daran gewohnt gewesen, praktisch allein über ihre Bereiche zu herrschen. Nun aber näherten sich ihnen die neuen Mandatsträger teilweise so stark an, daß sie ihnen Konkurrenz machten oder sie sogar ersetzten. Diese Spezialisierung bzw. Professionalisierung "im Sinne der Beherrschung einer technischen Kompetenz, des Besitzes eines bestimmten Fachwissens"12, wurde durch die berufliche Disponibilität der neuen Mandatsträger gefördert, die häufiger als zuvor im öffentlichen Sektor beschäftigt waren. Das Vorhaben, die Gebietskörperschaft transparenter, offener und sichtbarer zu machen, veranlaßte die Mandatsträger, in den Dienststellen stärker präsent sowie aktiv zu sein und durch ihre täglichen, vielfältigen Interventionen zu zeigen, daß sie eine dynamische Politik betrieben, die in einer Periode wirtschaftlicher Umstrukturierung stark gefragt ist. Wir haben diesen radikalen Stilwandel anhand der Untersuchung eines landesweiten Samples von mittleren Städten in den siebziger und achtziger Jahren bestätigen können ${ }^{13}$. Beigeordnete, die in der Regel abwesend oder unmotiviert waren - und die im übrigen noch nicht einmal über ein Büro im Rathaus verfügten, wo sie sich sowieso nur selten sehen ließen - , sind durch eine neue Generation von dynamischen, engagierten Mandatsträgern ersetzt worden: Sie sind vor Ort (in den Büros und Werkstätten, auf den Baustellen) präsent, verhandeln direkt mit den staatlichen Stellen (Ministerien, Außenstellen), haben ihre Zuständigkeitsbereiche im Griff und gelten in den Augen des städtischen Verwaltungspersonals häufig als "Lokomotiven". 


\section{Zentralisierung und Dezentralisierung}

Auf die Frage, ob Frankreich ein zentralisierter oder ein dezentralisierter Staat sei, ist man versucht, mit "Jein" zu antworten. Frankreich ist nach wie vor eines der am stärksten zentralisierten westlichen Länder, und zwar aufgrund der mehrere Jahrhunderte alten politischen und ideologischen Vereinheitlichung, die zu einer radikalen, alle Aktivitäten zwischen Paris und der Provinz erfassenden Hierarchisierung geführt hat. "Paris und die französische Wüste" lautet der berühmte Titel eines 1947 erstmals erschienenen und bis heute vielzitierten Buchs von Jean-François Gravier ${ }^{14}$. Mittlerweile unterzieht sich Frankreich jedoch einer Kur, um die Symptome der Pariser Hypertrophie zu bekämpfen - jener Krankheit, die dem Land in der Vergangenheit zu unvergleichlicher Macht verhalf, sich in den letzten Jahrzehnten aber immer mehr als Hemmschuh einer ausgewogenen Entwicklung erwiesen hat. Frankreich scheint sich heute auf dem Wege der Besserung zu befinden.

\section{Der Prozeß der institutionellen Reform}

Bis in die achtziger Jahre hinein war die rechtliche, technische und finanzielle Aufsichtsfunktion das traditionelle Mittel für den Staat, die Kommunen zu kontrollieren. Die Präfekten übten sie im Namen des Staates in den Departements aus. Andere staatliche Vertreter oder Verwaltungsinstanzen trugen ebenfalls zur Kontrolle und Zentralisierung des Territoriums bei, z.B. die Ingenieure der Tiefbauverwaltung (Ponts et Chaussées). Zahlreiche ländliche Kommunen sind immer noch weitgehend von der Fachkompetenz und den technischen Mitteln der zentralstaatlichen Stellen auf Departementsebene abhängig. Der Staatsrat (Conseil d'Etat), die höchste Instanz der Verwaltungsgerichtsbarkeit, intervenierte in der ersten Hälfte des 20. Jahrhunderts häufig, um lokale Initiativen einzuschränken oder zu verhindern. Von den dreißiger Jahren bis Ende der sechziger Jahre 
weitete die Zentralgewalt ihre Kontrolle der Lokalpolitik noch aus, insbesondere im Bereich der Raumordnung und Stadtplanung ${ }^{15}$.

Die Kommunen wurden allzu oft als vollkommen von den staatlichen Verwaltungsinstanzen abhängig dargestellt, womit der Grad der Zentralisierung sicherlich übertrieben wurde. Die Notabeln verfügten durchaus über Machtressourcen und unterhielten mit den Staatsbeamten auf territorialer Ebene Beziehungen der wechselseitigen Kontrolle. Die "wechselseitige Regulierung" und die Ämterhäufung sind als wesentliche Triebkräfte eines "gezähmten Jakobinismus" interpretiert worden, wo die anscheinend allmächtige Zentralgewalt sich mit den politischen Interessen der lokalen Mandatsträger arrangieren mußte ${ }^{16}$. Seit Ende der sechziger Jahre hat sich die staatliche Aufsicht in dem Maße gelockert, wie der Staat begriff, daß Anreize und Kompromisse gegenüber den Gebietskörperschaften effizienter waren als Anordnungen. Unter Präsident Valéry Giscard d'Estaing (1974-1981) unternahm die Regierung erste dezentralisierende Reformen, insbesondere durch die Lockerung der Kontrolle der Haushaltspolitik der Gebietskörperschaften (Einführungen von staatlichen Globalzuweisungen für die Gebietskörperschaften, Abschaffung der staatlichen Festsetzung der Gemeindesteuersätze, Lockerung einschränkender Subventionsregelungen).

Die Dezentralisierungsgesetze der achtziger Jahre - die François Mitterrand selbst als "wichtige Angelegenheit" seiner ersten Amtsperiode bezeichnete mögen zwar heute als grundlegend erscheinen, stellen jedoch lediglich eine Fortsetzung und Bestätigung der Emanzipationsbestrebungen der Gebietskörperschaften dar, die bereits einige Jahre vorher eingesetzt hatten. Das Gesetz vom 2. März 1982 führt drei bedeutende institutionelle Änderungen ein:

Abschaffung aller Vorabkontrollen, die durch im nachhinein durchzuführende Kontrollen ersetzt wurden, und zwar nicht nur 
hinsichtlich der Rechtmäßigkeit der Verwaltungsentscheidungen (Ausweitung der Rolle der Verwaltungsgerichte), sondern auch im Finanzwesen (Einrichtung von regionalen Rechnungshöfen);

- Übertragung der Exekutive der Departements- und Regionalräte, die bisher dem Präfekt oblag, auf die Präsidenten dieser Versammlungen;

- allgemeine Ermächtigung der Gebietskörperschaften, eigene wirtschaftliche Aktivitäten zu entfalten, was bisher strengen Vorschriften unterlag.

Die Gesetze vom 7. Januar und vom 22. Juli 1983 formulierten mehrere Prinzipien bezüglich des Kompetenztransfers zwischen Staat und Gebietskörperschaften:

- keine Gebietskörperschaft kann eine andere beaufsichtigen, weder in finanzieller, administrativer noch technischer Hinsicht;

- jeder Kompetenztransfer muß mit dem Transfer der entsprechenden Ressourcen einhergehen, d.h. der Ressourcen, die dem Staat zum Zeitpunkt des Transfers für die Ausübung dieser Kompetenz zur Verfügung standen;

- jeder der verschiedenen Kategorien von Gebietskörperschaften wird eine funktionale Rolle zugeschrieben: Städteplanung für die Kommune, Sozialhilfe und ländliche Infrastrukturversorgung für das Departement, wirtschaftliche Entwicklung (Berufsausbildung und Raumordnung) für die Region.

Diese grundlegenden Gesetzestexte sind durch eine Reihe anderer Reformmaßnahmen ergänzt worden, die insbesondere folgendes vorsehen: Verabschiedung eines allgemeinen Statuts, der für den gesamten öffentlichen Dienst auf territorialer (d.h. lokaler oder regionaler) Ebene gültig ist; Einführung der Direktwahl des Regionalrats; Schaffung von Bezirksräten in den drei größten französischen Städten Paris, Lyon und Marseille. 
Bei der Dezentralisierungsreform hat man es sorgfältig vermieden, heikle Probleme anzupacken: die unabdingbare Umgestaltung des Systems der Gemeindesteuern, das extrem ungerecht und archaisch ist; die offiziell befürwortete, aber niemals verwirklichte Zusammenlegung der 36000 Kommunen; die Verstärkung der Mechanismen, die die Partizipation der Bürger fördern. Weite Teile der staatlichen Verwaltung waren nicht sehr geneigt, die Neugewichtung der territorialen Kräfte zu unterstützen ${ }^{17}$. So empfahl der 1986 von der Regierung in Auftrag gegebene, von zwei Mitgliedern des Rechnungshofes verfaßte Feuilloley-Raynaud-Bericht zur kommunalen Finanzlage, in Anlehnung an die extrem zentralisierende Politik der britischen Regierung unter Margaret Thatcher eine direkte staatliche Kontrolle der lokalen öffentlichen Ausgaben zu schaffen. Diese Kontrolle sollte den Präfekten obliegen und es ihnen erlauben, die ausgabenfreudigsten Gebietskörperschaften zu sanktionieren, und zwar auf der Grundlage von nationalen Normen hinsichtlich Niveau und Entwicklung der Aufwendungen. 
Die trotz ihrer Unzulänglichkeiten umfassende Reform hat eine Reihe von Auswirkungen auf die französische Gesellschaft gezeitigt, die sowohl von der Bevölkerung als auch von den wichtigsten betroffenen Akteuren vor Ort (z.B. Mandatsträger, staatliche und territoriale Beamte, Vereine, Unternehmer) positiv bewertet werden. Allerdings hat sich ein Unbehagen ausgebreitet, das die Entwicklung ihres Amtes vielen lokalen Verantwortlichen einflößt und zum großen Teil auf die fehlende Abgrenzung der jeweiligen Aufgabenbereiche zurückzuführen ist. Die Aufteilung der Zuständigkeitsbereiche zwischen Staat, Kommunen, Departements und Regionen existiert zwar auf dem Papier (vgl. Tab. 1), ist aber in der Praxis sehr viel fluktuierender. In zahlreichen Sektoren kommt es zu Überlappungen (insbesondere durch die weit verbreitete Praxis der Mischfinanzierungen), was die Entscheidungskanäle extrem undurchsichtig werden läßt. Das entspricht einer Veränderung der öffentlichen Politik seit der Dezentralisierung; die Mischfinanzierungen oder die Zusammenarbeit von mehreren öffentlichen Akteuren auf territorialer Ebene sind Ausdruck der politischen Dominanz "großer Mandatsträger" auf einem durch eine Vielzahl von formal autonomen "kleinen Mandatsträgern" gekennzeichneten Markt ${ }^{18}$.

\section{Ist Frankreich heute dezentralisiert?}

Das wirtschaftliche und demographische Gewicht der Hauptstadt Paris und ihrer Region (Ile-de-France) hat sich in den letzten zwanzig Jahren sicherlich kaum verringert. Die Einwohnerzahl des städtischen Großraums Paris beträgt das 7,4-fache des nächstgrößeren Ballungsraums Lyon. Die Pariser Großköpfigkeit - die sich darin äußert, daß hier 26 Prozent der städtischen Bevölkerung leben - ist in Europa eine Ausnahmeerscheinung. Aber das Gerüst und die politisch-administrativen Ressourcen des übrigen französischen Territoriums haben an Bedeutung gewonnen. Es gibt zahlreiche Anzeichen eines tiefgreifenden Mentalitäts- und 
Strategiewandels bei den politischen, administrativen und ökonomischen Eliten. Der öffentliche Dienst der Gebietskörperschaften zieht heute oft die brillantesten und ehrgeizigsten Hochschulabsolventen an, weil er Möglichkeiten und Vorteile bietet, über die die Ministerien nicht mehr verfügen. Auch die Politiker tendieren dazu, sich auf ihre territoriale Basis $\mathrm{zu}$ besinnen, und geben der Konsolidierung ihrer lokalen Mandate und Beziehungen den Vorzug vor einer national ausgerichteten politischen Laufbahn.

Internationale Statistiken erlauben den Vergleich der Dezentralisierung des modernen Frankreichs mit anderen Industrieländern. So wurde beispielsweise die Bedeutung des kommunalen öffentlichen Sektors in Frankreich und in sieben anderen Ländern in den achtziger Jahren verglichen $^{19}$. Auf den ersten Blick scheint der öffentliche Dienst in Frankreich sehr viel zentralisierter zu sein. Bei näherer Betrachtung der Zahlen und ihrer Bedeutung wird jedoch ersichtlich, daß dieser Unterschied im wesentlichen mit dem Status des Personals eines Schlüsselsektors zusammenhängt: des Schulwesens. Das französische Erziehungsministerium ist eine riesige Administration mit einer Million Beschäftigten, d.h. der Hälfte des staatlichen Zivilpersonals. Fiele hingegen das Schulwesen in den Zuständigkeitsbereich der Gebietskörperschaften, wie dies in zahlreichen westlichen Ländern der Fall ist, dann wäre das Verhältnis umgekehrt: Die lokalen Verwaltungen würden nicht etwa 40 Prozent weniger Personen beschäftigen als der Staat, sondern 40 Prozent mehr. Zudem ist festzustellen, daß der relative Anteil der lokalen Bediensteten am Gesamtpersonal des öffentlichen Sektors zwischen den fünfziger und den achtziger Jahren deutlich zugenommen hat (von 14 auf 25 Prozent) und weiter steigt, da der Staat neue Aufgaben auf die Gebietskörperschaften überträgt. Innerhalb von sechzehn Jahren (19671983) hat sich die Anzahl der städtischen Bediensteten mehr als verdoppelt; sie ist von 394000 auf 836000 angestiegen. 
Über den rein quantitativen Vergleich der dezentralisierten Personal- und Finanzressourcen hinaus scheint es sinnvoller zu sein, einen globalen Untersuchungsrahmen heranzuziehen, der nicht nur einen, sondern drei grundlegende, für die Autonomie der Gebietskörperschaften ausschlaggebende Ziele berücksichtigt ${ }^{20}$ :

- Freiheit bzw. Handlungsspielraum der lokalen Akteure gegenüber den staatlichen Behörden;

- Partizipations- und Einflußchancen der Bürger vor Ort;

- Effizienz der Gebietskörperschaften.

Ausgehend von dieser Klassifizierung haben Hesse und Sharpe ${ }^{21}$ drei Modelle des local government ${ }^{22}$ erarbeitet, die sich durch eine unterschiedliche Gewichtung zwischen den drei Zielen auszeichnen. Während das angelsächsische Modell im wesentlichen auf den Zielen Freiheit und Effizienz beruht, bevorzugt das Modell französischer Prägung - auch als napoleonisches Modell bezeichnet - die politische Interessenvertretung, die auf einem extrem zersplitterten Territorium durch die Notabeln verkörpert wird, wohingegen das nordische Modell (Deutschland, Schweiz, Skandinavien) durch ein sehr gutes Gleichgewicht zwischen Handlungsspielraum der Gebietskörperschaften, demokratischem Imperativ und Effizienzstreben gekennzeichnet ist.

Die von Hesse und Sharpe vorgeschlagene Definition und Einschätzung des französischen Modells bedarf jedoch der Korrektur. Frankreich weicht seit den Dezentralisierungsreformen vom Ausgangsmodell ab. Die von Notabeln verkörperte politische Interessenvertretung - die Gefahr läuft, von ihnen vollkommen vereinnahmt zu werden ${ }^{23}$ - ist zwar nach wie vor eine französische Besonderheit. Gleichzeitig aber haben die Gebietskörperschaften noch nie über so viel Handlungsspielraum verfügt gegenüber dem Staat, dem man heute paradoxerweise eher vorwirft, 
unzureichend präsent zu sein und sich um die Politik auf territorialer Ebene nicht zu kümmern. Was die Effizienz anbelangt, so haben die Kompetenztransfers in zahlreichen Bereichen (insbesondere die Verwaltung der weiterführenden Schulen) bewiesen, daß die Gebietskörperschaften ihren Rückstand seit Anfang der achtziger Jahre rasch aufgeholt haben.

\section{Die zunehmende Komplexität des öffentlichen Handelns}

Seit etwa zwanzig Jahren ist insbesondere in den städtischen Ballungsgebieten eine Intensivierung der sozioökonomischen und politischen Konflikte zu beobachten, zu deren Bewältigung die existierenden öffentlichen Akteure immer weniger in der Lage zu sein scheinen.

\section{Die Tendenz zur Machtzersplitterung}

Die Gebietskörperschaften werden mit neuen Problemen wie der zunehmenden Bedeutung der sozialräumlichen Trennung, der wirtschaftlichen Entwicklung und des Umweltschutzes konfrontiert, die mehr denn je kooperative Verhaltensweisen erfordern. Gleichzeitig befinden sie sich in einem Prozeß der Zersplitterung, der dazu beiträgt, die Legitimität ihres Wirkens erheblich zu schwächen.

Die territoriale Zersplitterung spiegelt die Fragmentierung des Territoriums in zahlreiche Verwaltungseinheiten wider. Diese Balkanisierung führt zu einer Reihe von Funktionsstörungen: mangelnde Kontrolle bzw. mangelnde Betreuung der Entwicklung auf lokaler Ebene, unzureichende Ressourcen, Mangel an Führungsgeschick und Sachkenntnis, soziale und ethnische Spaltungen sowie die Tendenz zunehmender steuerlicher Disparitäten. 
Wie in anderen westlichen Ländern ist die Frage der territorialen Fragmentierung zu Beginn der neunziger Jahre in Frankreich wieder aufgetaucht. Viele Vertreter aus Politik, Wirtschaft und Verbänden machten sich für Zusammenschlüsse stark, die es erleichtern sollen, tausende von dahinsiechenden Landgemeinde ${ }^{24}$ zu reaktivieren und die Politik in den städtischen Regionen kohärenter zu gestalten. $\mathrm{Ob}$ es sich um die in Ballungsräumen erhobene Gewerbesteuer (taxe professionnelle d'agglomération) oder um die Neukonzeption der Stadt-Umland-Verbände (communautés urbaines) handelt: Die örtlichen Initiativen, Experimente oder globalen Reformvorhaben sind Ausdruck dieses neuen Willens, mit dem Tabucharakter zu brechen, der seit vielen Jahren jeder bedeutenden Veränderung der geographischen Gebietsaufteilung anhängt.

In diesem Zusammenhang muß die starke "Metropolisierung" der Wirtschafts- und Siedlungsentwicklung seit 1982 erwähnt werden, ein in vielen Ländern zu beobachtender Trend, der sich in einer steigenden Konzentration der Bevölkerung wie der wirtschaftlichen Produktion im Umfeld der bedeutendsten und wettbewerbsfähigsten Metropolen äußert. Während aber die Metropolen der anderen europäischen Länder in der Regel im Rückgang begriffen sind oder stagnieren, scheinen die großen französischen Ballungsräume (insbesondere im Süden) "sowohl die besondere Dynamik der südeuropäischen Städte als auch die Verbreitung des nordeuropäischen Modells der lockeren, ausgedehnten Metropole in Mitteleuropa"25 $^{25} \mathrm{zu}$ vereinen. Die Metropolisierung ist Ausdruck einer Verschärfung der Disparitäten hinsichtlich Dynamik und Wachstum, und zwar zwischen den Ballungsgebieten selbst wie zwischen den städtischen Polen und ihrem Hinterland. Von 1982 bis 1990 hatte die Region Ile-deFrance eine positive Arbeitsplatzbilanz von 371000 Arbeitsplätzen vorzuweisen, d.h. über die Hälfte der gesamtfranzösischen Bilanz, die sich auf 703000 Arbeitsplätze belief. Die Gebiete, die neben der Region Ile-deFrance in den achtziger Jahren die meisten, insbesondere hochwertigen 
Arbeitsplätze gewannen, sind das Städtenetz der Region Rhône-Alpes, der Ballungsraum entlang der Mittelmeerküste sowie Toulouse, Bordeaux, Rennes, Nantes und Straßburg. In dieser Spitzengruppe sind demnach die größten französischen Metropolen vertreten, deren Abstand zu den anderen Ballungsräumen sich vergrößert. Die wirtschaftliche Dynamik der Städte führt jedoch nicht notwendigerweise zur Dynamisierung ihres Hinterlands, im Unterschied zur industriellen Entfaltung der Jahre 1955-1975, deren Auswirkungen ganzen Regionen zugute kamen. Heute trägt das Wachstum dazu bei, die Disparitäten zwischen der Metropole und ihrer "Regionalperipherie" zu verschärfen.

Die Natur der Probleme hat sich indessen spürbar verändert. Die mehr oder weniger integrierten interkommunalen Kooperationsformen, die in den sechziger und siebziger Jahren geschaffen worden sind, haben ihre Unzulänglichkeit bei der Behandlung von immer stärker mit Konflikten belasteten Fragen unter Beweis gestellt, wie die Beispiele Städteplanung, wirtschaftliche Entwicklung, Verschärfung der sozioökonomischen Disparitäten, Steuerung der großen Kollektiveinrichtungen sowie Umweltschutz zeigen. So leiden die französischen Stadt-Umland-Verbände (communautés urbaines) darunter, daß ihre territoriale Zuständigkeit infolge der Ausbreitung der städtischen Ballungszonen nicht mehr realitätsgemäß ist. Vor allem aber fehlt es ihnen aufgrund ihrer Zusammensetzung (aus Delegierten der am Verband beteiligten Kommunen) an der die einzelnen Gemeinden übergreifenden Legitimität, die es ihnen erlauben würde, Konflikte und Blockierungen zu überwinden.

Die Tendenz zur Zersplitterung ist nicht nur territorialer, sondern auch funktionaler Natur. Die Regulierung der kommunalen Aktivitäten hängt mehr und mehr von einer Vielzahl von Instanzen ab, unter denen keine hierarchischen Beziehungen bestehen. Die negativen Auswirkungen dieser Fülle von unterschiedlichen Organisationen sind bekannt: steigende Kosten 
für die institutionenübergreifende Zusammenarbeit, geringere Transparenz des territorialen Systems für die Bürger und schließlich häufig eine Verschlechterung der Dienstleistungen.

Der Staat ist für diese Entwicklungen in hohem Maße verantwortlich. Denn die Dezentralisierung hat die Komplexität der Entscheidungskanäle erhöht, die aus der Häufung der Zuständigkeitsebenen resultiert. $\mathrm{Da}$ eine Minimaldefinition für die Verteilung der Aufgabenbereiche auf die Gebietskörperschaften fehlt, äußert sich täglich in der Zunahme von konkurrierenden, nicht koordinierten Initiativen, die dasselbe Territorium betreffen, und im exzessiven Rückgriff auf Mischfinanzierungen ${ }^{26}$. Die Beschaffenheit der Zuständigkeitsbereiche ist darüber hinaus undurchsichtig. Davon zeugt z.B. das unter den Franzosen weit verbreitete Unverständnis, was das Wirrwarr der Gemeindesteuern anbelangt ${ }^{27}$.

\section{Der neue Kontext des öffentlichen Handelns}

Die Tendenz zur funktionalen Zersplitterung der lokalen Selbstverwaltung verweist auf die Enthierarchisierung der Beziehungen zwischen den Gebietskörperschaften. Der Staat kann heute praktisch nicht mehr souverän handeln und seine Entscheidungen und politischen Maßnahmen den anderen öffentlichen Akteuren aufzwingen. Seine Interventionen bestehen weniger in Anweisungen als vielmehr in dem Bemühen, Impulse zu geben, zu beschleunigen oder zu koordinieren. Zu den wichtigsten Ursachen dieses tiefgreifenden Wandels gehören die objektiven Grenzen des Ausbaus des Wohlfahrtsstaats, die sich seit fünfzehn Jahren im rückläufigen Wirtschaftswachstum und in der daraus resultierenden Verknappung der nationalen öffentlichen Ressourcen äußern. Der Staat ist allmählich gezwungen worden, die auf supranationaler, vor allem auf EU-Ebene festgesetzten Prinzipien und Normen $\mathrm{zu}$ beachten. Die französische Regierung ist sehr darauf bedacht, ihre Prärogative zu schützen und sich in 
kritischen Bereichen wie der europäischen Regionalförderung gegen die Kompetenzansprüche der Regionen zu wehren ${ }^{28}$.

Die Zuständigkeit des Staates als "Gemeinwohlinstanz" stößt zudem auf die zunehmende Komplexität der öffentlichen Fragen. Die Probleme der benachteiligten Stadtviertel, die Reform des Schulsystems, der Ausbau der öffentlichen Verkehrsmittel, die Strukturierung von Wirtschaftszweigen, die Integration der ethnischen Minderheiten - dies alles sind politische Themen, die es erforderlich machen, Informationen, Ressourcen und Strategien zusammenzutragen. Innerhalb von nur wenigen Jahren ist auf der lokalen Ebene die führende Position, die der Staat lange Zeit für sich in Anspruch nahm und durch seine unterschiedlichen Instanzen auch wahrnehmen konnte, durch ein polyzentrisches System abgelöst worden, das nach einem neuen Gleichgewicht sucht. Bei zahlreichen Anlässen setzen sich Experten heute mehr oder weniger explizit für die Rückkehr eines starken Staates ein. Dieser Wunsch ist in Wirklichkeit häufig Ausdruck des Zurücksehnens nach einer Vergangenheit, als der Staat eine dominierende Rolle als Regulierungsinstanz spielte und gleichzeitig mit den Notabeln verhandelte.

Der neue Kontext des öffentlichen Handelns besitzt zumindest theoretisch den Vorteil einer stärkeren Machtteilung. Der Staat ist sich der Notwendigkeit bewußt, seine Beziehungen zu den Gebietskörperschaften stärker konsensorientiert zu gestalten. Sein Verhalten ist kooperativer, partnerschaftlicher geworden; er delegiert Aufgaben und konzentriert sich auf übergreifende Steuerungsfunktionen.

Die interne funktionale Zersplitterung ist zwar nicht neu, hat sich jedoch in den letzten Jahren unter dem Einfluß verschiedener, konvergierender Faktoren verstärkt. Neben der bereits erwähnten zunehmenden Komplexität der Probleme und dem Dezentralisierungsprozeß handelt es sich um eine 
Verschärfung der finanziellen Spannungen und um die neuen Regulierungsformen des öffentlichen Sektors auf territorialer Ebene.

Die Verschlechterung der wirtschaftlichen und finanziellen Rahmenbedingungen für die Gebietskörperschaften in den vergangenen zwei Jahrzehnten und die dadurch erzwungene Sparpolitik haben die soziopolitischen Veränderungen noch verstärkt. Die Bürger beurteilen ihre Vertreter zunehmend danach, ob es ihnen gelingt, die wirtschaftliche Leistungsfähigkeit und das allgemeine Wohlergehen zu verbessern. Die politischen Interventionen der Gebietskörperschaften hatten sich dieser Logik folgend stark ausgedehnt und differenziert in dem Maße, wie es neuen Herausforderungen $\mathrm{zu}$ begegnen galt: insbesondere in den Schlüsselbereichen des Schulwesens, der wirtschaftlichen Entwicklung und der Sozialhilfe. Angesichts der Schere zwischen steigenden Ausgaben und nunmehr rückläufigen Einnahmen sehen sich die politischen Mandatsträger heute höchst widersprüchlichen Wünschen der Bürger ausgesetzt, nämlich das Niveau der Dienstleistungen beizubehalten oder $\mathrm{zu}$ erhöhen und gleichzeitig die Ausgaben einzuschränken. In dieser Lage waren die Gebietskörperschaften zu problematischen Maßnahmen gezwungen: von der Einschränkung der Dienstleistungen bis hin zu Entlassungen und zur Schließung von Einrichtungen.

Dadurch werden territorialisierte Konflikte zugespitzt, weil sich der Wettbewerb zwischen den sozialen Gruppen um die knappen Ressourcen verschärft und die politisch-administrativen Verantwortlichen zwingt, ihre Prioritäten öffentlich darzulegen und stärker als zuvor unter den verschiedenen Interessenkategorien $\mathrm{zu}$ entscheiden. Das fördert das Auftreten radikaler Protestbewegungen gegen die bestehende politische Ordnung. Der Rechtsextremismus zehrt so von der wirtschaftlichen Rezession, die er als deutlichen Beweis für die Ineffizienz der pluralistischen Demokratie interpretiert sowie als Ausdruck der Unfähigkeit 
der traditionellen politischen Kräfte, "die wirklichen Probleme anzupacken". Auch die gesamte komplexe, empfindliche Dynamik der Beziehungen zwischen den verschiedenen Instanzen wird auf die Probe gestellt.

So versucht der Staat, sich der Verantwortung für die Umverteilung des Reichtums und für die Korrektur der Ungleichheiten, die er bisher als seine Aufgaben ansah, zu entziehen und diese auf die Gebietskörperschaften abzuwälzen. Letztere ziehen sich ihrerseits gegenüber den Anforderungen eines horizontalen Finanzausgleichs auf eine Position des lokalen Egoismus zurück.

Allerdings wäre es falsch zu behaupten, daß die Wirtschaftskrise bzw. die Rezession für alle Gebietskörperschaften dieselben Auswirkungen hat. Einige von ihnen wissen sich an die Verschlechterung ihres sozioökonomischen Umfeldes besser anzupassen als andere, wobei die Unterschiede in der Haushaltspolitik liegen. In Zeiten finanzieller Restriktionen suchen Mandatsträger und Kommunalverwaltungen zum Teil verzweifelt nach dem "goldenen Schnitt", der die Stabilität und den Wohlstand der Vergangenheit zurückbringt. Seit mehreren Jahren gibt es immer mehr Seminare, Bücher mit Wunderrezepten und Gurus des Verwaltungsmanagements, die Heilsbotschaften verkünden.

Zahlreiche Experten sehen die Einheit der Gebietskörperschaften heute am meisten durch die wachsende Privatisierung lokaler öffentlicher Dienste bedroht. Mit dieser Entwicklung ist eine mehr oder weniger radikale Veränderung der Funktionsweise der politisch-administrativen Systeme auf lokaler Ebene verbunden. Zum ersten Mal seit knapp hundert Jahren ist eine Zäsur in der Tendenz des kontinuierlichen Ausbaus des Interventionismus der Gebietskörperschaften festzustellen. Der "kommunale Sozialismus", der zu Anfang dieses Jahrhunderts triumphierte und die unabwendbare 
Entwicklung zum Fortschritt symbolisierte, findet heute kaum noch Anhänger: Er ist im Zuge der ideologischen Tendenzwende der achtziger Jahre durch den kommunalen Liberalismus verdrängt worden. Die Übertragung von Verantwortung auf Privatunternehmen entspricht in bestimmten Fällen einer offensichtlichen Kampfstrategie gegen einen öffentlichen Sektor, den man der Unbeweglichkeit, der mangelnden Anpassung an die sozialen Erwartungen und Bedürfnisse, der Schaffung von bürokratischen Privilegien und der finanziellen Verschwendung beschuldigt.

Konkrete Auswirkungen dieses neuen Trends sind allerdings bislang kaum zu spüren - im Gegenteil: Obwohl auch auf kommunaler Ebene immer wieder die Forderung nach einer Reduzierung des öffentlichen Sektors laut wird, hat das Personal der Gebietskörperschaften zwischen 1983 und 1993 weiterhin zugenommen ${ }^{29}$. So hat die Privatisierung zwar Stil und Inhalt der Managementpraxis verändert, scheint aber bisher die Balance zwischen öffentlichem und privatem Sektor nicht nennenswert beeinflußt zu haben. Insgesamt haben die durchgeführten Reformen den öffentlichen Interventionismus nicht spürbar eingeschränkt.

\section{Literaturauswahl}

Ashford, Douglas, British Dogmatism and French Pragmatism. CentralLocal Policymaking in the Welfare State, London: Allen and Unwin, 1982.

Balme, Richard / Garraud, Philippe / Hoffmann-Martinot, Vincent / Ritaine, Evelyne, Le territoire pour politiques: variations européennes, Paris: L’Harmattan (Coll. Logiques politiques), 1994. 
Becquart-Leclercq, Jeanne, Frankreich, in: Wehling, Hans-Georg (Hrsg.), Kommunalpolitik in Europa, Stuttgart: W. Kohlhammer Verlag, 1994, S. 63-101.

Duran, Patrice, France: Local Authorities in Transition, in: Hesse, Joachim Jens (Hrsg.), Local Government and Urban Affairs in International Perspective, Baden-Baden: Nomos, 1991, S. 429-461.

Gilbert, Guy / Delcamp, Alain (Hrsg.), La décentralisation dix ans après, Paris: Librairie générale de droit et de jurisprudence, 1993.

Grémion, Pierre, Le pouvoir périphérique, Paris: Seuil, 1976.

Hoffmann-Martinot, Vincent / Kjellberg, Francesco (Hrsg.), Décentraliser en France et en Norvège, Paris: Pédone, 1996.

Mabileau, Albert, Kommunalpolitik und -verwaltung in Frankreich. Das lokale System Frankreichs, Basel: Birkhäuser, 1996.

Mabileau, Albert / Sorbets, Claude (Hrsg.), Gouverner les villes moyennes, Paris: Pédone, 1989.

Uterwedde, Henrik, Kommunen in Frankreich und Deutschland. Eine Einführung/Communes en France et en Allemagne. Une introduction, Bonn: Gemini, 1991.

\footnotetext{
1 Zum Verwaltungssystem vgl. grundlegend Bruno Rémond / Jacques Blanc, Les collectivités locales, Paris: Presses de la Fondation nationale des sciences politiques/Dalloz, 1989; ferner das seit 1981 erscheinende Jahrbuch der
} 
Gebietskörperschaften, zuletzt: Groupement de recherches sur l'administration locale en Europe/ GRALE (Hrsg.), Annuaire des collectivités locales 1997, Paris: Litec, 1997. Knappe, übersichtliche Einführungen bieten Pierre Sadran, Le système administratif français, Paris: Montchrestien, 1997²; Emmanuel Vital Durand, Les collectivités territoriales en France, Paris: Hachette, 1994.

${ }^{2}$ Zahlen für 1994. Zum staatlichen Personal gehören jedoch weder die Beschäftigen der Post, von France Télécom und der nationalen öffentlichen Einrichtungen noch die Lehrkräfte der vertraglich an den Staat gebundenen Privatschulen. Vgl. Direction générale des collectivités locales, Les collectivités locales en chiffres. Edition 1996, Paris: Direction générale des collectivités locales, 1997.

${ }^{3}$ Vgl. Dokumentation.

${ }^{4}$ Pierre Grémion, Ansätze politischer und administrativer Dezentralsierung in Frankreich, in: Deutsch-Französisches Institut (Hrsg.), Bausteine zum Systemvergleich, Band 1, Gerlingen: Bleicher, 1980, S. 104; vgl. ders., Le pouvoir périphérique, Paris: Seuil, 1976.

${ }^{5}$ Vgl. Jean-Pierre Worms, Le préfet et "ses" notables, in: Sociologie du Travail, (1966) 3, S. 249.

${ }^{6}$ Weniger als der Staat (1 553 Mrd.) oder die Kommunen (433 Mrd.), aber wesentlich mehr als die Regionen (67 Mrd. Francs).

${ }^{7}$ Vgl. Pierre Sadran, Ambivalences de la région, in: Guy Gilbert / Alain Delcamp (Hrsg.), La décentralisation dix ans après, Paris: Librairie générale de droit et de jurisprudence, 1993, S. 395-399.

${ }^{8}$ Gesonderte Regelungen für die Territorialverwaltung gelten z.B. in den überseeischen Departements und Territorien, in Korsika, im Elsaß, im Departement Moselle (Lothringen) sowie in den drei Metropolen Paris, Lyon und Marseille.

${ }^{9}$ Yves Mény, La République des fiefs, in: Pouvoirs, (1992) 60, S. 17.

10 Ein britischer Autor betonte bereits zu Beginn des Jahrhunderts die Macht des französischen Bürgermeisters: "Wenn der Bürgermeister ein energischer Mensch ist, kann er fast ein Stadtdiktator sein." (Percy Ashley, Le pouvoir central et les pouvoirs locaux, Paris: Marcel Girard, 1921, S. 90).

${ }^{11}$ Vgl. Philippe Garraud, Profession: homme politique. La carrière politique des maires urbains, Paris: L'Harmattan, 1989.

12 Albert Mabileau / Pierre Sadran, Administration et politique au niveau local, in: Francis de Baecque / Jean-Louis Quermonne (Hrsg.), Administration et politique sous la Cinquième République, Paris: Presses de la Fondation nationale des sciences politiques, 1982, S. 269.

${ }^{13}$ Vgl. Vincent Hoffmann-Martinot, Les services comme enjeu, in: Albert Mabileau / Claude Sorbets (Hrsg.), Gouverner les villes moyennes, Paris: Pédone, 1989, S. 53.

${ }^{14}$ Jean-François Gravier, Paris et le désert français, Paris: Le Portulan, 1947.

${ }^{15}$ Vgl. Douglas Ashford / Jean-Claude Thoenig (Hrsg.), Les aides financières de l'Etat aux collectivités locales en France et à l'étranger, Paris: Litec, 1981.

16 Vgl. Michel Crozier / Jean-Claude Thoenig, La régulation des systèmes organisés complexes. Le cas du système de décision politico-administratif local en France, in: Revue Française de Sociologie, XVI (1975) 1, S. 3; Pierre Grémion, Le pouvoir périphérique, Paris: Seuil, 1976.

17 Vgl. Jack Hayward / Edward Page, Instruments de mise en oeuvre des politiques gouvernementales et relations centre-périphérie en Grande-Bretagne et en France, in: Politiques et Management Public, (1985) 3, S. 81. 
${ }^{18}$ Vgl. Guy Gilbert / Jean-Claude Thoenig, Les cofinancements entre collectivités publiques. Une étude prototype. Rapport intermédiaire de recherche à la DATAR, Paris, März 1997, S. 171 f.

19 Vgl. Vincent Hoffmann-Martinot, Les employés municipaux et les politiques du personnel: une comparaison de huit pays, in: GRALE, Annuaire des collectivités locales 1991, Paris: Litec, 1991, S. 53.

${ }^{20}$ Wir beziehen uns auf Lawrence J. Sharpe, Theories and Values of Local Government, in: Political Studies, Vol. XVIII (1970) 2, S. 153.

${ }^{21}$ Vgl. Joachim Jens Hesse / Lawrence J. Sharpe, Local Government in International Perspective: Some Comparative Observations, in: Joachim Jens Hesse (Hrsg.), Local Government and Urban Affairs in International Perspective, Baden-Baden: Nomos, 1991.

22 Der im Französischen benutzte Begriff "gouvernement local" ist aus dem angelsächsischen Begriff "local government" abgeleitet und hat im Deutschen keine direkte Entsprechung, könnte aber mit "lokale Selbstverwaltung" umschrieben werden.

${ }^{23}$ Sie sollen die einzigen großen Sieger der Dezentralisierung sein, so die Analyse von Jacques Rondin, Le sacre des notables, Paris: Fayard, 1985.

24 Jacques Palard zufolge ist die sehr hohe Anzahl von kommunenübergreifenden Zweckverbänden eher Ausdruck eines "halben Mißerfolgs": Communes rurales, décentralisation et coopération. Gestion territoriale ou développement local, in: Guy Gilbert / Alain Delcamp (Hrsg.), La décentralisation dix ans après, Paris: Librairie générale de droit et de jurisprudence, 1993, S. 217-230.

${ }^{25}$ Alain Sallez / Pierre Vérot, Les villes en question, in: Alain Sallez (Hrsg.), Les villes, lieux d'Europe, Paris: Editions de l'Aube, 1993, S. 147-178.

${ }^{26}$ Vgl. Albert Mabileau, Kommunalpolitik und -verwaltung in Frankreich. Das "lokale System" Frankreichs, Basel: Birkhäuser, 1996, S. 53.

${ }^{27}$ Ergebnisse einer Umfrage von Crédit local en France (CLF)-SOFRES (Le Monde vom 19. November 1996, S. 13, 31, und vom 4. Dezember 1996, S. 12).

${ }^{28} \mathrm{Zu}$ diesem "Marmorkuchen" Europäische Union/Staat/Region vgl. Jacques Palard, Les fonctions de la politique régionale européenne: développement économique, cohésion sociale ou intégration politique, in: Vincent Hoffmann-Martinot / Francesco Kjellberg (Hrsg.), Décentraliser en France et en Norvège, Paris: Pédone, 1996, S. 59-77.

${ }^{29}$ Die Steigerungsrate beträgt 18,3 Prozent gegenüber einem Bevölkerungsanstieg von 4,9 Prozent - trotz eines gleichzeitigen Rückgangs des Personals der Departementsverwaltungen in Höhe von 12,6 Prozent als Ergebnis der Transfers von den Departements auf den Staat; vgl. Direction générale des collectivités cocales, Les agents des collectivités territoriales, Paris: Direction générale des collectivités locales, 1996, S. 97. 